\title{
FUNCTION OF THE RUMEN
}

$\mathrm{T}$ HE 123rd meeting of the Nutrition Society was held at the Royal Veterinary College, London, under the chairmanship of Dr. D. P. Cuthbertson, director of the Rowett Research Institute, on March 7, when a symposium on "Rumen Function" took place. In his opening remarks the chairman described the theme as one of great fundamental and practical interest where the microbiologist, biochemist, physiologist, animal husbandman, veterinarian, and members of the feeding stuffs trade could meet and learn much through the exchange of knowledge and concepts.

Dr. C. C. Balch (National Institute for Research in Dairying, Shinfield) introduced the subject by describing the structure of the ruminant stomach and the movement of its contents. In the course of its development the ruminant stomach has achieved an efficient microbial digestion of plant fibres and also of practically all other plant constituents, and with this goes the syntheses of microbial polysaccharide and protein and also certain vitamins The structure of the four portions of the ruminant stomach which emerge from the embryonic stomach - the rumen, reticulum, omasum and abomasumwas described. The abomasum with its typical gastric secretory function corresponds to the stomach of non-ruminants ; in very young ruminants it is as large as the rumen.

Co-ordinated movements of the omasum, reticuloomasal orifice, reticulum and rumen can be recorded pneumatically or seen radiographically. These were described by Dr. Balch.

During eating, the food boli are deposited in the anterior rumen, where they rapidly merge. By the end of a meal of hay most of the new food is to be found tightly packed in the dorsal sac as a relatively dry, fibrous layer, whereas the ventral region will contain a layer of more fluid digesta. The reticulorumen never empties, and, in fact, in the stall-fed animal rarely contains less than half its capacity. The amounts found in grazing animals are considerably less. The amount of food given, and especially the amount of hay, is a major factor influencing the length of time residues remain in the rumen : there is a slight tendency for concentrates and succulents to pass out of the reticulo-rumen more rapidly than roughages. According to Gordon, the onward passage of particles of hay is greatest during rumination. The transfer of digesta to the omasum is a continuous process.

Dr. B. H. Howard (Rowett Research Institute, Aberdeenshire), in his contribution on the meta. bolism of carbohydrates by rumen bacteria, first described the various techniques which have been used: (1) in vivo studies; (2) 'artificial rumen' preparations ; (3) washed cell suspensions ; (4) direct microscopic observation including use of fluorescing antibodies; (5) pure culture; and (6) 'enrichment culture' experiments.

Cellulose is the most important of the polysaccharides fermented in the rumen, and xylan occurs in close association with it. Most of the rumen cellulolytic and xylan-decomposing bacteria are very strict anaerobes and some require earbon dioxide and/or rumen liquor. Cellulose yields mainly acetic, lactic and succinic acids in pure culture fermentations, and xylan gives fatty acids, from formic to butyric. Mixed rumen organisms, on the other hand, convert both substances mainly into varying proportions of acetic and propionic acids.

The main rumen starch-fermenter is Streptococcus bovis, chains of cells of which can be seen attached to decomposing stareh grains. It produces only an $\alpha$-amylase, and lactic and acetic acids are the chief fermentation products. The same organism will also ferment the oligo- and poly-saccharides containing fructose, found in grass and root crops.

Rumen micro-organisms show a striking capacity to synthesize polysaccharides either internally as iodophilic deposits of 'starch-like' material, or externally as capsules or slime-layers. It is doubtful if the material leaving the rumen to enter the omasum contains any significant amount of digestible carbohydrate of microbial origin.

Lactic and succinic acids added to the rumen are quickly decomposed, yielding mainly propionic acid. Rumen acetic and butyric acids are interconvertible. Formic acid is rapidly decomposed in the rumen to carbon dioxide and hydrogen, which further react to give methane; some five rumen-volumes per day arise from this reaction normally.

In his paper on the utilization of protein by the dairy cow, Dr. M. J. Head (National Institute for Research in Dairying) said that as a result of the action of the ruminal micro-organisms, the value of the nitrogenous moiety of the feed to the dairy cow is not so dependent on its amino-acid structure or its proportion of true protein as is the case with a single-stomached animal. The ammonia produced during the fermentation is either utilized for the growth of organisms which are later digested to give nutrients of high biological value, or is absorbed through the rumen wall into the blood-stream and largely wasted. This loss has not been sufficiently considered in evaluating protein feeds for ruminants.

The classical assessment of the biological value of a protein is complicated in the case of a ruminant by the fermentation in the rumen, as has been shown by the work of Chalmers and Synge. The greater the loss of ammonia from a protein, the greater the significance to the animal of the amino-acid structure of the feed protein residues leaving the rumen unattacked.

A comparison of the various oil-cake residues normally used as protein sources for dairy cows on the basis of daily in vivo rumen ammonia concentration curves did indicate differences between the cakes. A similar classification of these protein sources could be made on the basis of the proportion of their total nitrogen soluble in $1 M$ sodium chloride solution.

While theoretically the most desirable type of protein for a ruminant is one of low solubility in the rumen and yet of high biological value for a singlestomached animal, two further questions need consideration with reference to practical feeding. First, is the dairy cow normally rationed sufficiently stringently for any of these theoretical improvements to be seen to advantage; and secondly, may it not be more economic to feed a theoretically wasteful protein source if it still supplies cheaper units of biological value ?

In discussing lipids in relation to rumen function, Dr. G. A. Garton (Rowett Research Institute) pre- 
faced his paper by a consideration of the nature of dietary lipids. The lipids present in feeding stuffs such as grass, hay, silages and concentrates were considered with special reference to the $C_{18}$ polyunsaturated fatty acids (linoleic and linolenic) which form a large part of the total fatty acid content.

Events in the rumen were considered first with reference to the hydrogenation of unsaturated fatty acids, which occurs to a considerable extent under the influence of the rumen bacteria. This results in the production, inter alia, of stearic acid which, following intestinal absorption and deposition, may well account for the characteristic 'hardness' of ruminant depot fats.

Recent findings on the hydrolysis of triglyceride fats in the rumen by a bacterial lipase wore then outlined. These demonstrate that most of the fatty acids entering the rumen as glycerides reach the abomasum and small intestine in the free state-a situation markedly different from that in monogastric animals in which partial lipolysis takes place in the small intestine. The fermentation of the liberated glycerol (to yield propionic acid) was briefly discussed.

The ruminant does not appear likely to derive any of the so-called 'essential' fatty acids from the lipids of its rumen bacteria. Thus it appears that, if the ruminant requires 'essential' fatty acids, they must be of dietary origin and must also escape hydrogenation in the rumen.

In his contribution on milk composition in relation to rumen metabolism, Dr. J. A. F. Rook (National Institute for Research in Dairying) indicated that the observations of Powell in 1938-41 provided convincing evidence that the fine grinding of the entire roughage in a ration or, alternatively, a considerable reduction in roughage intake and an increase in the concentrate feed, reduced the percentage and yield of fat without affecting total milk production. This specific effect on fat secretion has been fully confirmed by Balch, Balch, Bartlett, Cox and Rowland at Shinfield. Milk of unusually low fat content was observed by MeClymont to result from cows grazing on young herbage or fed on kale, concentrates and limited amounts of hay or straw, that is, diet short of roughage. The operative factor is the physical condition of fibrousness rather than tho presence of fibre.

The depression in the milk fat secretion of cows on low roughage diets has invariably been associated with a change in fat composition. Milk fat in the cow and other ruminants is distinguished from the milk fat of other species, and from animal depot fats in general, by a relatively high content (up to 20 per cent on a molar basis) of short-chain $\left(\mathrm{C}_{2}-\mathrm{C}_{10}\right)$ acids, and when a reduction in fat secretion occurs the proportion of these acids is correspondingly reduced. Some reduction in the weights of higher saturated (largely palmitic and stearic) and also unsaturated (largely oleic) acids secreted is also observed.

Additions of sodium acetate (500-1,500 gm./day) or acetic acid have produced an almost complete recovery in the fat content of milk, but individual animals have failed occasionally to show a response to sodium butyrate. It is held to be the change in the production of acetic and propionic acids that has generally been responsible for the depression in milk fat synthesis.

The role of the protozoa was outlined by Dr. Margaret Eadie (Rowett Research Institute) in her paper on some aspects of rumen ciliate protozoa.
According to Dr. Eadie, the basic nature of the association of rumen ciliates with rumen function is a complex one still not clearly understood, but their study is valuable because of the one justified generalization: that certain species of ciliates have been found in the rumen of all normal adult ruminants so far examined.

In general, diet appears to have the greatest effect upon the eiliate population, and variations are so large that no acceptable average figure can be given nor can the ciliate protein contribution be accurately assessed. This may be as high as 20 per cent of the total host requirement, and qualitative studies have indicated that the ciliate protein is of higher nutritional value than the rumen bacterial protein.

Comparisons of faunated and non-faunated animals have so far shown no serious effects on those free from ciliates, but it has yet to be ascertained whether advantage from a ciliate population would appear under conditions of stress. In recent work in collaboration with Dr. Preston at the Rowett Institute, experiments are in progress to determine, by variation of the roughage content of the weaning ration, how soon ciliates may be permanently established in early-weaned calves and whether this is advantageous.

Numerically the oligotrich organisms, particularly Entodinium spp., make up the largest proportion of most populations. So far, the holotrichous organisms, which are fewer in number, have been most closely studied and the information of the biochemical and nutritional properties of these organisms suggests that equally interesting information should accrue from similar studies of the oligotrich ciliates.

In his paper on the treatment and prevention of bloat with anti-foaming agents, Mr. C. S. W. Reid (Plant Chemistry Division, Department of Scientific and Industrial Researeh, Palmerston North, New Zealand, at the Physiological Laboratory, Cambridge) pointed out that if stable foams are generated during fermentation, the elimination of gas from the rumen is impeded and bloat (tympanites) may result. His paper reviewed work carried out in New Zealand on the use of anti-foaming agents in the treatment and prevention of acute bloat in grazing dairy cattle. Administration of foam-breaking substances-fats and oils, certain detergents and other surface-active materials but not silicones-was the only consistently useful treatment found. Materials at present recommended for field use are peanut oil and emulsified ruminant tallow in doses of 120-150 gm. fat. Antifoaming agents were also effective when given prophylactically. Pasture spraying proved to be a reliable control measure and has been used on New Zealand farms over four seasons. It has also been notably successful in preventing bloat on irrigated pastures in Australia.

Detectable anti-foaming activity is present in the rumen contents and can be shown to be affected by the activity of the micro-organisms. However, apart from indicating the probable complexity of the situation, observations so far have not revealed obvious correlations between lipid intake, antifoaming activity and bloating.

Dr. A. T. Phillipson (Rowett Research Institute) in his paper on the rumen in relation to the animal considered first the extent of digestion in the reticulum and rumen. He pointed out that several estimates have been made of the extent of digestion in the reticulum and rumen which are based on the lignin ratio and which suggest that the quantity of food constituents disappearing ranges from 43 to 83 per 
cent in cattle and that a similar but rather narrower range has been found in sheep. Most of the material disappearing is carbohydrate. It is more difficult to assess the extent of disappearance of nitrogencontaining materials as the secretions augment the nitrogen of the food, but it appears that considerable losses occur from the rumen in both cows and sheep and presumably these represent the absorption of ammonia. However, losses in the intestine are more extensive when compared with the apparent digestibility of nitrogen for the whole alimentary tract.

Estimates of fatty acid production in the rumen show that they are quantitatively important. The recent work of Armstrong and Blaxter at the Hannah Dairy Research Institute indicates that at levels of feeding up to maintenance the value of the mixture of short-chain fatty acids to the animal as a source of energy is fairly constant irrespective of the quantities of each individual acid absorbed. For production purposes, rations that favour the formation of propionic and butyric acids should be more valuable than those that favour the production of acetic acid. The proportions of propionic and butyric acids increase as the concentrate part of the ration increases in cattle. In particular, starch-rich food increases the proportion of propionic acid. As yet, very little is known of the peptic and intestinal phases of digestion.

In the second part of his paper, Dr. Phillipson dealt briefly with the absorptive capacity of the intestinal tract. One of the conditions of terrestrial life is the necessity of conserving water and sodium, and the ruminant is unusually good at carrying out these two tasks.

The quantities of water and sodium secreted in the saliva alone are far greater than the quantities excreted in the faeces. The contribution made by other digestive juices still needs investigation and is likely to be considerable. Both sodium and water are known to be absorbed from the rumen and omasum, while there is no doubt that absorption also occurs along the remainder of the tract, particularly in the large intestine. It is of particular interest that in conditions of sodium depletion the sodium content of the saliva is lowered and its place is taken by potassium; there is also a diminution of the volumes secreted. A study of the rates of turnover of body constituents such as water, sodium and other elements and nitrogen in various circumstances may help in the end to understand why occasionally things go wrong.

The final paper of the symposium was by $\mathrm{Mr}$. J. K. D. Dow (Unilever, Ltd.) on the application of recent scientific information through the feeding. stuffs industry.

Studies on the absorption of fatty acids in the rumen, and the connexion with hormone metabolism, indicate dietary means of regulating milk production and quality and of curing conditions such as ketosis. Chemical tests enable processed protein materials to be classified according to the needs of the animals for which they are best suited. The feeding-stuffs industry has already made use of research into the vitamin and mineral requirements of ruminants. Further research on the effect of these on roughage utilization is awaited.

Recent work on antibiotic supplements for calves has indicated results which are not applicable through the industry in the present stage of legislation. This case demonstrates the wide view which is demanded of the research worker to-day, particularly when some manufacturers may depend entirely on published scientific evidence in formulating new feeding stuffs. However, it is doubtful whether fundamental research workers have adequate facilities for testing their results under practical conditions. This problem offers a profitable field for consideration by the feeding-stuffs industry, because co-operation between the research worker and the manufacturer is essential to the efficient functioning of the industry and, ultimately, will ensure that the farmer reaps the fullest benefit.

\section{NUCLEAR FUEL CYCLES}

$\mathrm{T}$ HE Institute of Physics, in collaboration with the British Nuclear Energy Conference, held a symposium on "Nuclear Fuel Cycles" at the Institution of Civil Engineers, Westminster, on January 22 and 23 . The symposium was opened by the president of the Institute of Physics, Sir George Thomson, who welcomed some 250 members and visitors, including delegates from eight European countries. $\mathrm{He}$ stressed the importance of fuel cycles in relation to the large nuclear power stations now under construction in the United Kingdom. These would prove competitive with coal-fired stations only if their uranium fuel were employed in the most efficient manner.

The symposium was divided into three sessions, the first of which considered the basic physics of long-term reactivity changes and nuclear fuel cycles, the second was concerned with the optimization of fuel cycles, and the third session discussed relevant operational problems. The chairman of the first session was Dr. J. V. Dunworth (Atomic Energy Research Establishment, Harwell). The first paper presented was that by Dr. H. Rose and Mr. J. J. Syrett (Atomic
Energy Research Establishment, Harwell) on "Long. term Reactivity Changes", which deseribed the basic physical processes occurring as natural or slightly enriched uranium is irradiated in large graphitemoderated thermal reactors. The importance of these ehanges was emphasized since, together with considerations of metallurgical damage, they would determine the irradiation-level at which the fuel must be replaced. The overall reactivity-changes with irradiation are determined mainly by the small net difference between the large changes arising from the burn-up of uranium-235 and the formation of plutonium-239, making the problem unusually sensitive to all the relevant nuclear data. Furthermore, the plutonium isotopes formed in the irradiated uranium have large resonances in their fission and capture cross-sections at low neutron energies. The prodiction of their rates of reaction with neutrons therefore demands a detailed knowledge of neutron spectrum conditions to an extent unnecessary in assessing the initial reactivity of the reactor. For these reasons, present predictions rely considerably upon integral measurements which are made on irradiated fuel 